\title{
High precision gravitational self-force calculations and post-Newtonian implications
}

\author{
Bernard F Whiting ${ }^{* \dagger}$ \\ University of Florida, Gainesville, United States \\ E-mail: bernardephys.ufl.edu
}

This talk is based on $[1,2,3]$. Until less than 10 years ago, post-Newtonian $(\mathrm{PN})$ analysis was the only possible systematic method for obtaining gravitational waveforms corresponding to binary inspiral. However, these were cut-off before the merger, until the recent availability of direct results from numerical relativity computations, which could include the complete merger and ring-down phase of the orbital evolution. Unfortunately these calculations are not yet of sufficient precision to strenuously test PN methods intrinsically. By contrast, the gravitational self-force approach has become capable of advancing to extremely high precision, and of thereby testing most of the various techniques used in PN calculations. Although restricted to the extreme-massratio limit, self-force calculations are now able to verify both the methods and results of PN work, and even of extending it. In fact, as will be demonstrated, they now have high enough precision to be able to determine new coefficients analytically.

Frontiers of Fundamental Physics 14 - FFP14,

15-18 July 2014

Aix Marseille University (AMU) Saint-Charles Campus, Marseille

\footnotetext{
* Speaker.

${ }^{\dagger}$ With Luc Blanchet, Guillaume Faye, John Friedman and Abhay Shah
} 


\section{Introduction}

General relativity is a theory of the non-linear, dynamical interaction between matter and geometry. Despite the difficulties inherent in understanding such a theory, we do know that it introduces physical consequences which are not manifest in the Newtonian theory of gravity. However, there is still much that we do not know about the full implications of general relativity. Various attempts to understand the theory have focussed on analytical, approximative and fully numerical approaches. Figure 1 represents both these various methods, and their respective domains of applicability. Most recently, a network of gravitational wave detectors has been developed worldwide, specifically so that we can learn more about matter in the strong field regime of general relativity.

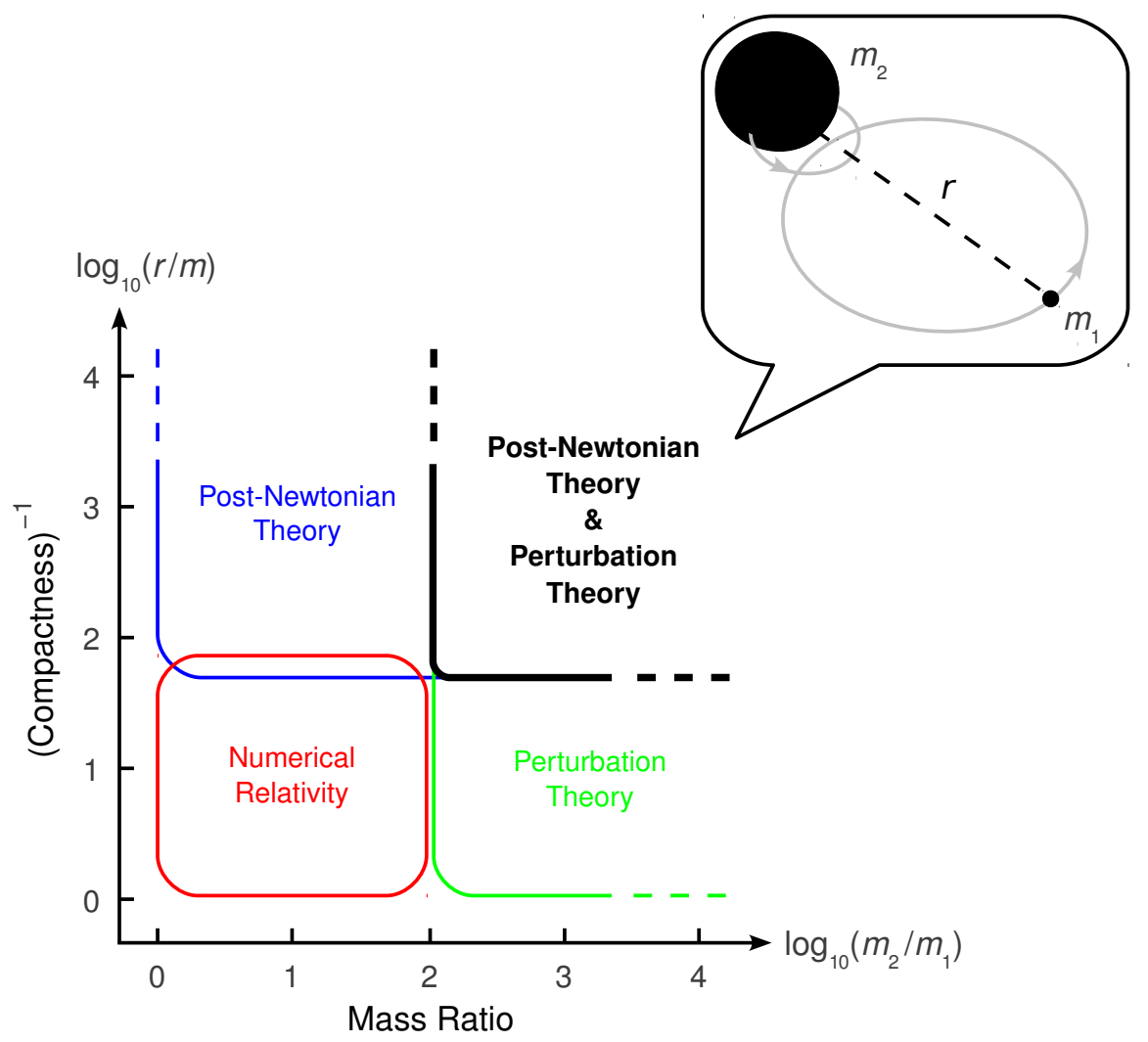

Figure 1: Different analytical approximation schemes and numerical techniques are used to study compact binary systems, depending on the mass ratio $m_{1} / m_{2}$ and the orbital velocity $(v / c)^{2} \sim$ $m / r_{12}$, where $m=G\left(m_{1}+m_{2}\right) / c^{2}$. Post-Newtonian theory and perturbation theory can be compared in the slow motion regime ( $v \ll c$ equivalent to $r_{12} / m \gg 1$ for circular orbits) of an extreme mass ratio $\left(m_{2} / m_{1} \gg 1\right)$ binary.

For almost a decade now, numerical relativity has been able to evolve compact, binary systems through the final stages of their inspiral, into their merger phase, and to follow the rapid ring-down of the resulting single compact body, which may well be a black hole. Today, the best waveforms from comparable-mass inspiraling binaries are obtained from a combination of analytical and numerical approaches, and are used as templates in gravitational wave searches. However, these 
calculations work best for systems of equal mass, and fail for binary systems with an extreme mass ratio, since very different timescales are required for compact bodies with very different masses.

In this work, we concentrate on distinct approximation methods, namely, the post-Newtonian scheme, which can deal with celestial bodies in slow motion $(|\mathbf{v}| \ll c)$, and the self-force approach which has been developed to treat a binary system with an extreme mass ratio, $m_{1} m_{2} \ll\left(m_{1}+m_{2}\right)^{2}$, in the fully relativistic regime. More specifically, we will compare a PN expansion for the extreme mass ratio inspiral (EMRI) problem with a combination of numerical and analytical results from self-force calculations.

This comparison serves several purposes. First, because the two approaches are so different and conceptually independent, any agreement between them can be taken as a vindication of the methods used in both. Second, the self-force calculations will be carried out to such a high numerical precision that analytic results will be obtainable with practical certainty. Third, this will occur at a sufficiently high post-Newtonian order, that it will be possible to predict analytic coefficients not yet obtained by PN calculations and to give them explicitly.

\section{What is the self-force problem?}

Under gravity, a test particle moves along a geodesic. If the particle has finite (but relatively small) mass, its motion is influenced by the field it, itself, creates - the particle modifies the geometry through which it moves by a self-interaction effect known as the self force (see Figure 2). The self force has both conservative and dissipative effects. A change in the relationship between circumference and period of an orbit is conservative, while the evolution of the orbital frequency as a result of radiation reaction (shown in Figure 3a) is a dissipative effect. Unfortunately, the "circumference" of an orbit is not a physical observable, in that it is not gauge invariant, while the frequency and period, as seen from infinity, are physically observable, and are gauge invariant.

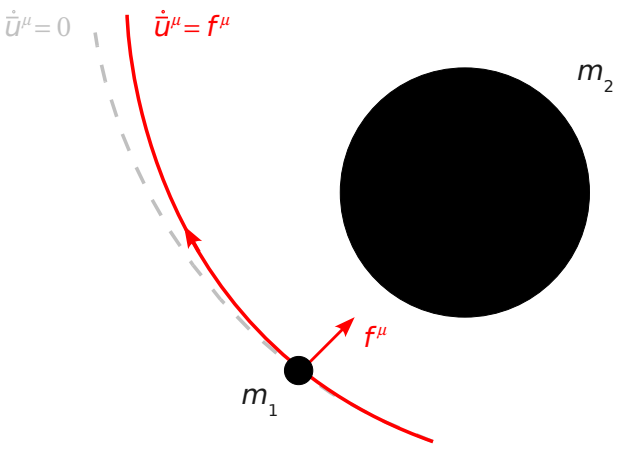

Figure 2: The self force results in motion which is not geodesic in the background geometry, but in a modified, smooth geometry.

\subsection{Why is it a "problem"}

General relativity has the additional feature that the center of mass of an extended body is also not uniquely definable, so even the orbit itself of an extended body is not really well defined. A common computational aid has been to define massive bodies as point particles, but in this singular limit, the geometry becomes non-differentiable. By a loose analogy with electromagnetism, we might expect the self force to be given by the gradient of some field, but in general relativity the geometry is the field, and the gradient does not exist if the field is non-differentiable. So, this computational aid requires the additional introduction of a regularization scheme, as a means of enabling a calculation the result of which will be finite. 


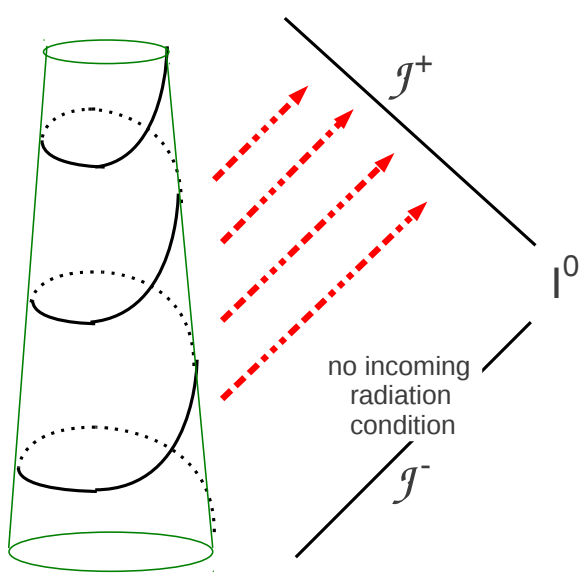

Physical situation

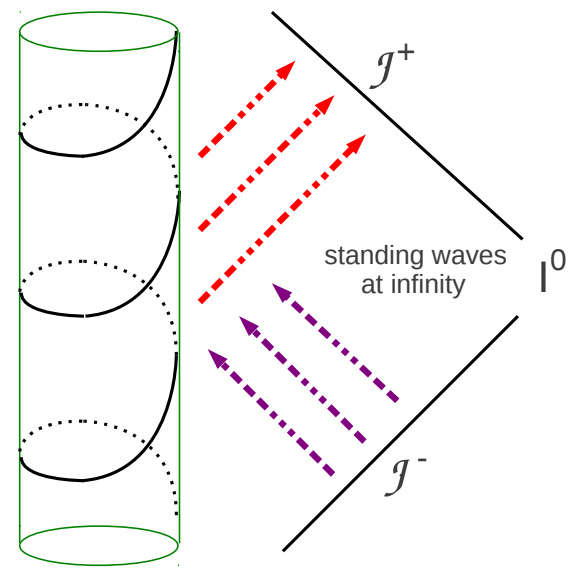

Situation with the HKV

(b) Standing waves at infinity

(a) No incoming radiation at past null infinity

Figure 3: In the physical situation, the orbit evolves as shown in (a). Incoming radiation balances the outward flux at infinity in (b). It ensures there is enough symmetry to have a helical Killing vector, to which the particle's four-velocity is related by the gauge invariant scale factor $U^{t}(\Omega, v)$.

Hence, the self force problem arises for EMRI binaries and is a combination of a technical difficulty associated with the physical description of the self force as well as a computational difficulty associated with a practical simplification of the physical description for calculational purposes. It is further exacerbated by the fact that the self force itself is not gauge invariant - in fact there is a modified, smooth geometry in which the orbital motion can be described as geodesic [4] - but its determination is effectively essential for computing orbital evolution, for which the changing frequency as observed from infinity is truly a physical observable. Thus, the long term objective of all self force calculations is to obtain the frequency (or phase) and amplitude of the gravitational waveform emitted from a specified physical system.

The calculations we shall consider will both use the point particle simplification. However, they have very different methods for extracting a smooth manifold and obtaining finite results from the singular limit. In this situation, to compare two quantities which are not gauge invariant and calculated by entirely different means can be fraught with difficulty. Our method of dealing with this is to make one further change to the problem being specified with the result that a gauge invariant quantity can be identified. The one change is that, instead of allowing the orbit to start our circular and evolve, we effectively balance the outgoing radiation with an equal amount of incoming radiation, whereby the orbit remains unchanged throughout the calculation (Figure 3b).

\subsection{Brief historical background}

The modern approach to the self force problem was developed by Dirac in 1938 for the electromagnetic problem [5]. To work in a curved space-time, as required in general relativity, further geometric developments were required, and these were presented by DeWitt and Brehme in 1960 [6], but it was not until the late 1990's that a coherent formulation for the gravitational self force 
was given by two separate groups, leading to what is now known as the MiSaTaQuWa equation $[7,8]$. Subsequent contributions have included: the determination of a smoothly perturbed metric in which the self force motion can be described by a geodesic [4]; the realization that $\ell=0$ and $\ell=1$ contributions played an essential role in the regularization of the gravitational self force [9]; the introduction of effective sources which allow avoidance of integration in the singular neighborhood of the assumed point source [10,11]; and, description of a suitable scaling limit permitting a more rigorous explanation of the MiSaTaQuWa equation [12], to name a few.

Until the late nineties, post-Newtonian calculations offered the only estimate of self force effects, even though such calculations are expected to be unreliable once the orbit enters the strong field regime. Beginning with the work of Mino, Sasaki and Tanaka [7], a well defined scheme for computing self force effects was developed, using a perturbative approach based on full general relativity. Since then, rigorous methods of characterizing contributions to the self force in a nonsingular way have been identified, and geometric perturbations to first order in the mass ratio of an EMRI binary have been obtained for both circular and elliptical orbits with both non-spinning and even spinning sources. In this report, our focus will be on calculations which allow comparison between the PN and perturbative SF approaches.

\section{Post-Newtonian and perturbative self force comparisons}

For the purposes of the comparison presented here, we shall use the Schwarzschild space-time for the large mass, $m_{2}$, by itself and, in fact, could effectively use a Schwarzschild space-time also for the small mass, $m_{1}$. In the presence of the small mass, the full space-time no longer has the symmetry of the space-time associated with the larger mass by itself but, for a circular orbit, there is a residual helical Killing vector in the combined geometry. The four-velocity of the small mass is necessarily tangent to this Killing vector and we can use the constant of proportionality as a gauge invariant quantity to be calculated as a function of the orbital frequency. We will adopt a coordinate system in which the helical Killing vector can be represented everywhere as $K^{\mu} \partial_{\mu}=\partial_{t}+\Omega \partial_{\phi}$, where $\Omega$ is the orbital frequency. The constant of proportionality for the normalized four-velocity $U^{\mu}$ can then be taken as $U^{t}$, and it will be investigated as a function of both $\Omega$ and the mass ratio $q=m_{1} / m_{2}$ (or alternatively, the symmetric mass ratio, $v$, given be $\left.v=m_{1} m_{2} /\left(m_{1}+m_{2}\right)^{2}\right)$. The gauge invariance of $U^{t}$ was first identified by Detweiler, who also realized that $1 / U^{t}$ determines the redshift of a photon, emitted from particle $m_{1}$, when the photon is observed on the orbital-axis at very large distance [13]. Thus, $1 / U^{t}$ is sometimes referred to as Detweiler's redshift variable.

Both self-force and post-Newtonian calculations require the specification of a near zone and a far zone. In both cases these zones must overlap, and matched asymptotic expansions are used to do matching in the overlap region. In the PN case, the interior zone extends outside the source in the near zone, where the PN field is matched to a multipole expansion which is defined all over the exterior zone and reaches into the far field domain, with the far zone treated as being asymptotically Minkowskian. In the SF case, the near zone can be thought of as centered around the small mass: Near the small mass, the larger mass imposes a weak tidal distortion of its gravitational field, while far from the small mass, its effect can be viewed as a weak perturbation of the Schwarzschild geometry of the large mass. Matching is required, in order to determine the conservative adjustment 

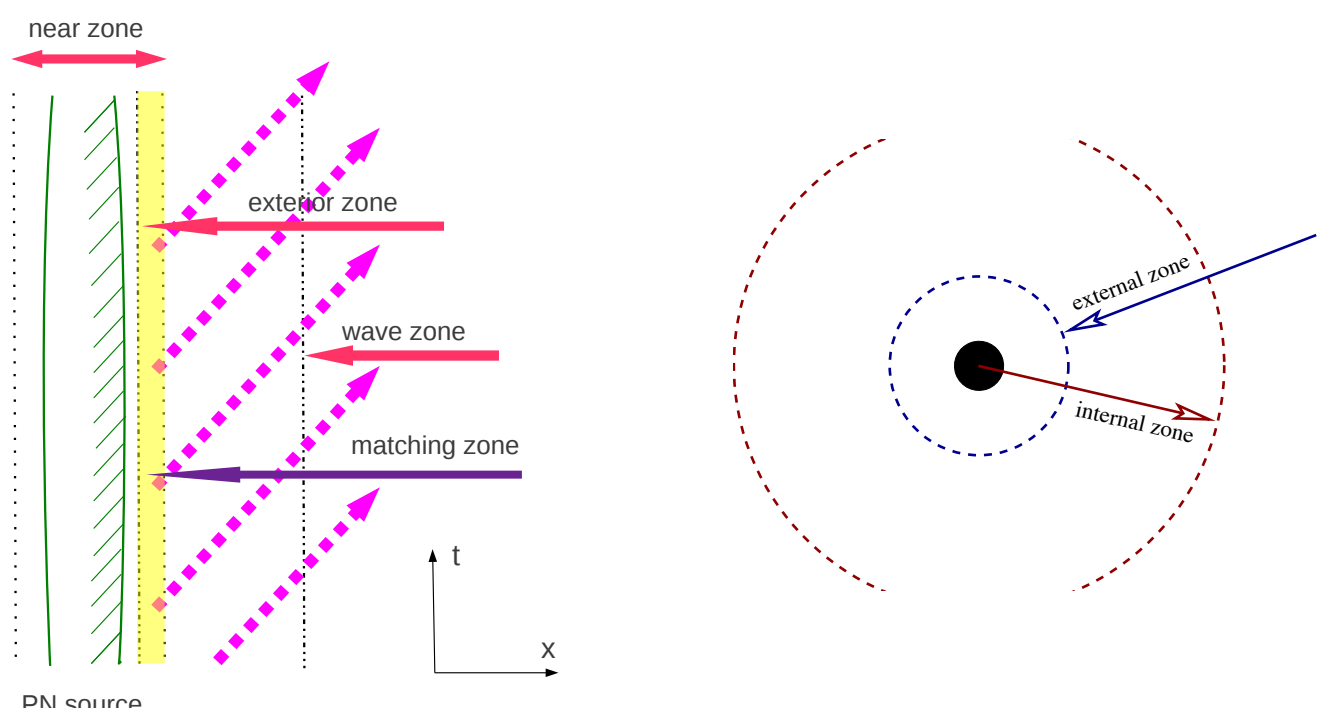

PN source

(a) PN matching is done in the yellow (shaded) region (b) SF matching is done between the dotted spheres

Figure 4: The post-Newtonian (a) and self-force (b) calculations require interior and exterior zones. In both cases these must overlap. Matched asymptotic expansions are used to do the matching.

to the small mass orbit which is required by the self force effect. The ensuing equation of motion arises from seeking a simple multipole (body centered) description near the small mass.

\subsection{How is high precision enabled?}

Until recently, all self force calculations based on the Schwarzschild geometry as background have been performed either by working directly with the coupled, linearized, Einstein field equations ( $\delta G_{\mu \nu}=8 \pi G_{N} c^{-4} \delta T_{\mu \nu}$ ), or by using the Bardeen-Press equation (for perturbations of the Weyl tensor) [14], or the Regge-Wheeler [15] and Zerilli equations [16, 17]. Calculations have been carried out in both the time domain and the frequency domain, with the latter generally affording better accuracy, though not necessarily speed. For the most accurate results, this has required developing a series expansion of sufficient precision near the horizon and near infinity, and then using numerical integration of a radial ODE to find solutions everywhere. Such was the basis of early comparisons between SF and PN results, which were generally limited to double precision numerically $[13,18]$, but was enough to reveal the explicit appearance of logarithm terms, which were later confirmed by direct post-Newtonian calculation [19, 20].

The representation of solutions in terms of new, convergent, globally valid, series expansions - first developed more than 15 years ago by Mano, Suzuki and Tagasugi (MST) [21], but applied to these comparison problems for $U^{t}(\Omega, v)$ only in the last few years [1] - has dramatically changed this situation. In the case of the perturbed Weyl scalar, $\psi_{0}$, decomposed in terms of ${ }_{2} Y_{\ell, m_{\phi}}(\theta, \phi)$ on 
the sphere, the relevant radial expansions can be given as:

$$
\begin{aligned}
& R_{H}=e^{i \varepsilon x}(-x)^{-2-i \varepsilon} \sum_{n=-\infty}^{\infty} a_{n} F(n+v+1-i \varepsilon,-n-v-i \varepsilon,-1-2 i \varepsilon ; x), \\
& R_{\infty}=e^{i z} z^{v-2} \sum_{n=-\infty}^{\infty}(-2 z)^{n} b_{n} U(n+v+3-i \varepsilon, 2 n+2 v+2 ;-2 i z) .
\end{aligned}
$$

where $x=1-r c^{2} /\left(2 G_{N} m_{2}\right), \varepsilon=2 G_{N} m_{2} m_{\phi} \Omega / c^{3}, z=-\varepsilon x, r$ is the radial coordinate in the Schwarzschild geometry of the large mass, the $F$ are the ordinary hypergeometric functions of Gauss, the $U$ are the confluent hypergeometric functions introduced by Francesco Tricomi, and $v$ is (not the symmetric mass ratio) chosen such that (both) series converge as $n$ approaches both $\pm \infty$.

The $v$ required for convergence of the sums above can be determined as an expansion in $\ell$ and $\varepsilon$. Sufficient terms must be retained to ensure the accuracy of eqns (3.1) and (3.2) to any specified number of digits (which currently run into the thousands). For example, for $\varepsilon$ sufficiently small, corresponding to orbits such that $r_{12} / m \sim 10^{30}$ (see Fig. 1 caption for definition of $m$ ), we find $n_{\max } \simeq-n_{\min } \simeq 35$ gives several hundred digits of accuracy. Remarkably, this accuracy is sufficient to determine several coefficients in the expansion for $U^{t}$ exactly. This is possible because, at any finite order in the PN expansion for $U^{t}$, the coefficients have only a limited range of complexity, being a sum of such terms as $1, \pi, \gamma$ (the Euler gamma constant), and $\ln p$ (where $p$ may be a prime: $2,3,5$, etc.), all with rational coefficients composed of integers of finite extent. It is that finiteness which allows us to extract analytically exact coefficients from results of finite accuracy [22].

\section{Recent Achievements}

At the time Detweiler [13] first made the comparison between SF and PN calculations, PN waveforms for compact binaries were known to $3.5 \mathrm{PN}$, but the regularized metric required for the computation had not yet been calculated at 3PN order. This was subsequently found and published in joint work with Blanchet and Le Tiec [18]. At that time we were not immediately able to fit the numerical SF data for higher order PN coefficients, until it was realized that logarithm terms should be included at 4PN and all higher integer orders. With that realization, and the computation of the 4PN and 5PN logarithm coefficients, we were able to predict numerical values of the PN coefficients up to 7PN with $1 \%$ or better accuracy. That is where results stood for some time.

With the introduction of the MST formalism for representing gravitational perturbations, and by including enough terms in the various series, it became possible to calculate $U^{t}$ to at least 225 digits of accuracy [1], for circular orbits up to $10^{30} G c^{-2} m_{2}$ in the Schwarzschild radial coordinate. Somewhat unexpectedly, it was found that half-integer terms were required at 5.5PN order and beyond in order to fully represent the available data. This implies the existence of a corresponding $5.5 \mathrm{pN}$ term in the expansion of the energy of a binary system, which was subsequently confirmed by explicit PN calculation [23, 2] and, with Blanchet and Faye, these coefficients were algebraically confirmed up to 7.5PN order [3]. By realizing that a subset of PN coefficients would be rational numbers or products of $\pi$ and a rational, we had been able to obtain new, exact analytic, expressions for several additional PN coefficients, including the coefficient of the logarithm cubed at 10PN order [1]. Additionally, we were able to predict the value of a number of other coefficients up to 
10.5PN order with high numerical precision. Since this talk was given, some of these have now ben algebraically confirmed up to $9.5 \mathrm{PN}$ order [24, 25].

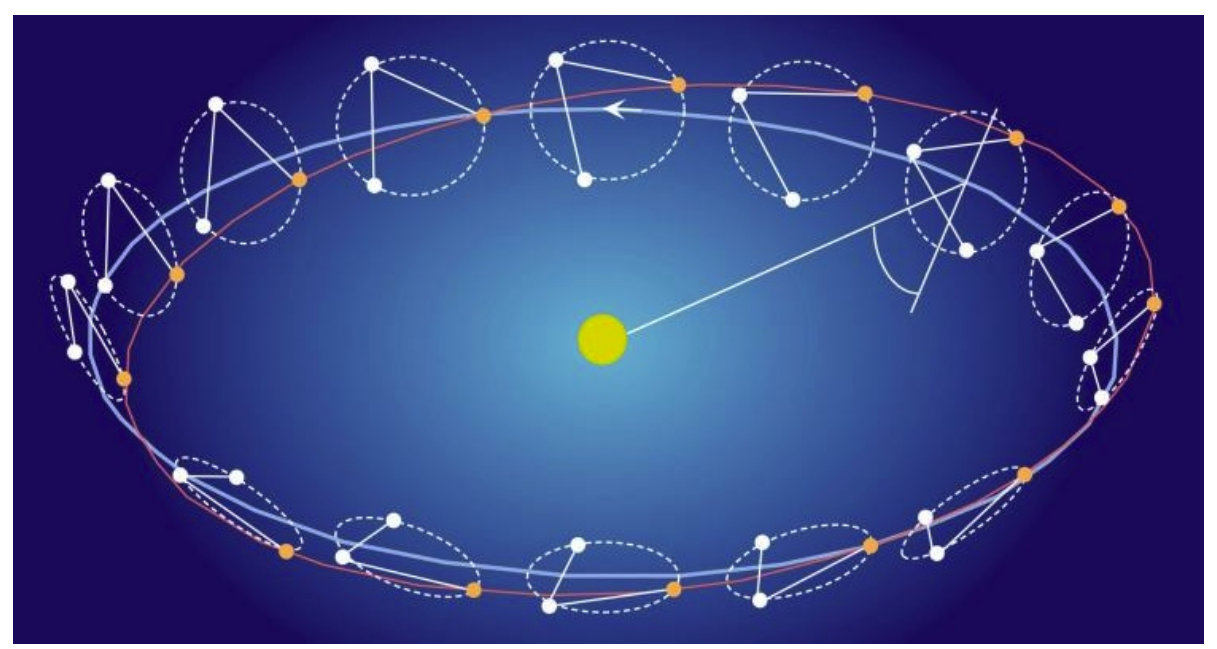

Figure 5: eLISA would be a space-borne experiment to detect gravitational waves from EMRIs. Three spacecraft in a triangle rotate as they orbit the Sun following the Earth with a $60^{\circ}$ lag.

\section{Impact on gravitational waveforms}

As mentioned in the previous paragraph, demonstrated in [26], and discussed more extensively in [27], high order PN coefficients in $U^{t}$ at some order in $v$ allow one to determine PN coefficients in the PN expansion of the gravitational binding energy at the same order. Even though these coefficients have now been determined for very small values of $v$, they are valid throughout the whole range $v=m_{1} m_{2} /\left(m_{1}+m_{2}\right)^{2} \leq 1 / 4$. This has lead to the complete non-spinning effectiveone-body (EOB) metric at linear order in the mass ratio [28]. The EOB formalism was introduced by Damour and colleagues to simplify the description of a two-body system [29, 30] and now plays a dominant role in the computation of waveforms for gravitational wave detection using groundbased instruments.

The EMRI systems to which self-force calculations pertain are typically composed of a solarmass-scaled body and a supermassive black hole, such as might occur in the center of a galaxy. The orbital periods associated with these systems are in the sub- $\mathrm{Hz}$ range and not accessible from the ground. Instead, space-borne instruments such as eLISA (conceptualized in Figure 5) have been proposed to detect gravitational waves from EMRI systems. One feature of the eLISA design is that detectable signals from EMRI sources should take of the order of a year to pass through the eLISA waveband. With the high precision of phase needed to track these systems accurately, first order (in $v$ ) self force calculations will be insufficient. Hence, there is a growing need, and effort, to develop self force calculations to second order, which we now briefly describe.

\section{The advent of second order}

In the last few years three different approaches have been outlined for proceeding to second 
order. These have been proposed by Detweiler [31], Gralla [32] and Pound [33] and appeared within a few months of each other. All are rather formal and do not contain sufficient detail for one to proceed with an explicit calculation. More recently, to help ameliorate this situation, Pound has, within a second-order self-force formalism, derived a second-order generalization of Detweiler's redshift variable [13], which provides a gauge-invariant measure of conservative effects on quasicircular orbits [34]. Once his or a similar scheme has been implemented, its results may be used to determine high-order terms in post-Newtonian theory and parameters in effective-one-body theory.

Pound's proposal for $U^{t}$ at second order is given by:

$$
\begin{aligned}
\tilde{U}^{t}(\Omega, q) & =\frac{1}{\sqrt{1-3\left(m_{2} \Omega\right)^{2 / 3}}}\left\{1+\frac{1}{2} \varepsilon h_{u_{0} u_{0}}^{\mathrm{R} 1}\right. \\
& \left.+\varepsilon^{2}\left[\frac{1}{2} h_{u_{0} u_{0}}^{\mathrm{R} 2}+\frac{3}{8}\left(h_{u_{0} u_{0}}^{\mathrm{R} 1}\right)^{2}-\frac{1}{6 \Omega^{2}}\left(1-3\left(m_{2} \Omega\right)^{2 / 3}\right)\left(\hat{F}_{1 r}\right)^{2}\right]+O\left(\varepsilon^{3}\right)\right\}
\end{aligned}
$$

where $h_{u_{0} u_{0}}^{\mathrm{R} 1}$ and $h_{u_{0} u_{0}}^{\mathrm{R} 2}$ represent the first and second order regularized metric perturbations contracted with the background velocity vector, $\hat{F}_{1 r}$ is the (time symmetric) radial component of the first order self force and $\varepsilon$ counts the powers of $q$ involved. PN theory already has an expression beyond 3PN at second order in $v=q /(1+q)^{2}$. It is interesting to ask if these two formulations are defined under the same conditions? Perhaps only numerical comparison will be able to tell us.

\section{Summary}

Post-Newtonian analysis, perturbative self force calculations, and full non-linear numerical relativity, all play a rôle in exploring gravitational waveforms from binary sources. By contrast with comparable, solar-mass-scale sources of gravitational waves which should be detectable from the ground, EMRIs have very different local timescales and should be evident for 100,000+ orbits. Post-Newtonian methods can calculate to all orders in $v=m_{1} m_{2} /\left(m_{1}+m_{2}\right)^{2}$ but requires $v / c$ to be small. Perturbative self-force calculations manage the highly relativistic regime, but each order in $m_{1} / m_{2}$ must be handled separately. Both methods have to regularize singularities and must treat radiative effects separately. Self-force calculations have extended the post-Newtonian expansion for the gravitational binding energy by several PN orders at first order in the mass ratio. PostNewtonian results will help identify the right comparison to be carried out at second SF order.

\section{Acknowledgments}

The author acknowledges his co-authors Luc Blanchet, Steven Detweiler, John Friedman, Guillaume Faye, Abhay Shah and Alexandre Le Tiec, without whom the entire work described here would not have been initially carried out. Throughout the time involved, this work was supported by NSF Grants PHY-0555484, PHY 0855503, PHY 1205906 and PHY 1314529 to the University of Florida. Sabbatical support from the CNRS through the IAP, where part of this work was carried out is acknowledged, as well as support from the French state funds managed by the ANR within the Investissements d'Avenir programme under Grant No. ANR-11-IDEX-0004-02. 


\section{References}

[1] A. G. Shah, J. L. Friedman, and B. F. Whiting, Finding high-order analytic post-Newtonian parameters from a high-precision numerical self-force calculation, Phys.Rev. D89 (2014), no. 6 064042, [arXiv:1312.1952].

[2] L. Blanchet, G. Faye, and B. F. Whiting, Half-integral conservative post-Newtonian approximations in the redshift factor of black hole binaries, Phys.Rev. D89 (2014), no. 6 064026, [arXiv:1312.2975].

[3] L. Blanchet, G. Faye, and B. F. Whiting, High-order half-integral conservative post-Newtonian coefficients in the redshift factor of black hole binaries, Phys.Rev. D90 (2014), no. 4044017 , [arXiv:1405.5151].

[4] S. L. Detweiler and B. F. Whiting, Selfforce via a Green's function decomposition, Phys.Rev. D67 (2003) 024025, [gr-qc/0202086].

[5] P. A. Dirac, Classical theory of radiating electrons, Proc.Roy.Soc.Lond. A167 (1938) 148-169.

[6] B. S. DeWitt and R. W. Brehme, Radiation damping in a gravitational field, Annals Phys. 9 (1960) 220-259.

[7] Y. Mino, M. Sasaki, and T. Tanaka, Gravitational radiation reaction to a particle motion, Phys.Rev. D55 (1997) 3457-3476, [gr-qc/ 9606018 ].

[8] T. C. Quinn and R. M. Wald, An Axiomatic approach to electromagnetic and gravitational radiation reaction of particles in curved space-time, Phys.Rev. D56 (1997) 3381-3394, [gr-qc/9610 053 ].

[9] S. L. Detweiler and E. Poisson, Low multipole contributions to the gravitational selfforce, Phys.Rev. D69 (2004) 084019, [gr-qc/ 0312010$].$

[10] I. Vega and S. L. Detweiler, Regularization of fields for self-force problems in curved spacetime: Foundations and a time-domain application, Phys.Rev. D77 (2008) 084008, [arXiv: 0712.4405 ].

[11] L. Barack and D. A. Golbourn, Scalar-field perturbations from a particle orbiting a black hole using numerical evolution in 2+1 dimensions, Phys.Rev. D76 (2007) 044020, [arXiv: 0705 . 3620 ].

[12] S. E. Gralla and R. M. Wald, A Rigorous Derivation of Gravitational Self-force, Class.Quant.Grav. 25 (2008) 205009, [arXiv:0806.3293].

[13] S. L. Detweiler, A Consequence of the gravitational self-force for circular orbits of the Schwarzschild geometry, Phys.Rev. D77 (2008) 124026, [arXiv: 0804 . 3529].

[14] J. Bardeen and W. Press, Radiation fields in the schwarzschild background, J.Math.Phys. 14 (1973) 7-19.

[15] T. Regge and J. A. Wheeler, Stability of a Schwarzschild singularity, Phys.Rev. 108 (1957) 1063-1069.

[16] F. J. Zerilli, Effective potential for even parity Regge-Wheeler gravitational perturbation equations, Phys.Rev.Lett. 24 (1970) 737-738.

[17] F. Zerilli, Gravitational field of a particle falling in a schwarzschild geometry analyzed in tensor harmonics, Phys.Rev. D2 (1970) 2141-2160.

[18] L. Blanchet, S. L. Detweiler, A. Le Tiec, and B. F. Whiting, Post-Newtonian and Numerical Calculations of the Gravitational Self-Force for Circular Orbits in the Schwarzschild Geometry, Phys.Rev. D81 (2010) 064004, [arXiv:0910.0207]. 
[19] L. Blanchet, S. L. Detweiler, A. Le Tiec, and B. F. Whiting, High-Order Post-Newtonian Fit of the Gravitational Self-Force for Circular Orbits in the Schwarzschild Geometry, Phys.Rev. D81 (2010) 084033, [arXiv:1002.0726].

[20] L. Blanchet, S. Detweiler, A. Le Tiec, and B. F. Whiting, High-Accuracy Comparison between the Post-Newtonian and Self-Force Dynamics of Black-Hole Binaries, Fundam.Theor.Phys. 162 (2011) 415-442, [arXiv:1007.2614].

[21] S. Mano, H. Suzuki, and E. Takasugi, Analytic solutions of the Teukolsky equation and their low frequency expansions, Prog.Theor.Phys. 95 (1996) 1079-1096, [gr-qc/ 9603020 ].

[22] N. K. Johnson-McDaniel, Taming the post-Newtonian expansion: Simplifying the modes of the gravitational wave energy flux at infinity for a point particle in a circular orbit around a Schwarzschild black hole, Phys.Rev. D90 (2014), no. 2 024043, [arXiv: 1405.1572 ].

[23] D. Bini and T. Damour, High-order post-Newtonian contributions to the two-body gravitational interaction potential from analytical gravitational self-force calculations, Phys.Rev. D89 (2014), no. 6 064063, [arXiv:1312.2503].

[24] D. Bini and T. Damour, Analytic determination of the eight-and-a-half post-Newtonian self-force contributions to the two-body gravitational interaction potential, Phys.Rev. D89 (2014), no. 10 104047, [arXiv:1403.2366].

[25] D. Bini and T. Damour, Detweiler's gauge-invariant redshift variable: analytic determination of the nine and nine-and-a-half post-Newtonian self-force contributions, arXiv:1502.02450.

[26] A. Le Tiec, L. Blanchet, and B. F. Whiting, The First Law of Binary Black Hole Mechanics in General Relativity and Post-Newtonian Theory, Phys.Rev. D85 (2012) 064039, [arXiv:1111.5378].

[27] A. Le Tiec, E. Barausse, and A. Buonanno, Gravitational Self-Force Correction to the Binding Energy of Compact Binary Systems, Phys.Rev.Lett. 108 (2012) 131103, [arXiv:1111.5609].

[28] E. Barausse, A. Buonanno, and A. Le Tiec, The complete non-spinning effective-one-body metric at linear order in the mass ratio, Phys.Rev. D85 (2012) 064010, [arXiv:1111.5610].

[29] A. Buonanno and T. Damour, Effective one-body approach to general relativistic two-body dynamics, Phys.Rev. D59 (1999) 084006, [gr-qc/9811091].

[30] T. Damour, P. Jaranowski, and G. Schaefer, On the determination of the last stable orbit for circular general relativistic binaries at the third postNewtonian approximation, Phys.Rev. D62 (2000) 084011, [gr-qc/0005034].

[31] S. Detweiler, Gravitational radiation reaction and second order perturbation theory, Phys.Rev. D85 (2012) 044048, [arXiv:1107.2098].

[32] S. E. Gralla, Second Order Gravitational Self Force, Phys.Rev. D85 (2012) 124011, [arXiv:1203.3189].

[33] A. Pound, Second-order gravitational self-force, Phys.Rev.Lett. 109 (2012) 051101, [arXiv:1201.5089].

[34] A. Pound, Conservative effect of the second-order gravitational self-force on quasicircular orbits in Schwarzschild spacetime, Phys.Rev. D90 (2014), no. 8 084039, [arXiv: 1404.1543 ]. 